\title{
Racial Disparities in Survival Among Advanced Non- Small Cell Lung Cancer Patients First-Treated with The Combination of Paclitaxel and Platinum
}

\section{Chenyang Zhao}

Center for Drug Clinical Evaluation, Shanghai University of Traditional Chinese Medicine https://orcid.org/0000-0003-0153-2677

\section{Fengli Wang}

Shanghai University of Traditional Chinese Medicine

Jihan Huang

Shanghai University of Traditional Chinese Medicine

\section{Yinghua Lv}

Shanghai University of Traditional Chinese Medicine

\section{Fang Yin}

Shanghai University of Traditional Chinese Medicine

Hongxia Liu

Shanghai University of Traditional Chinese Medicine

\section{Qingshan Zheng}

Shanghai University of Traditional Chinese Medicine

Lujin Li ( $\sim$ lilujin666@163.com )

Shanghai University of Traditional Chinese Medicine

\section{Research}

Keywords: Paclitaxel, Platinum, Advanced NSCLC, Overall Survival, Modeling and simulation

Posted Date: September 3rd, 2020

DOl: https://doi.org/10.21203/rs.3.rs-67603/v1

License: (c) (i) This work is licensed under a Creative Commons Attribution 4.0 International License.

Read Full License 
1 Racial Disparities in Survival Among Advanced Non-

2 6 17

\section{Small Cell Lung Cancer Patients First-Treated with The Combination of Paclitaxel and Platinum}

Chenyang Zhao ${ }^{1}$, Fengli Wang ${ }^{1}$, Jihan Huang ${ }^{1}$, Yinghua Lv ${ }^{1}$, Fang Yin ${ }^{1}$, Hongxia Liu ${ }^{1}$, Qingshan Zheng ${ }^{1}$, Lujin $\mathrm{Li}^{1}$

${ }^{1}$ Center for Drug Clinical Evaluation, Shanghai University of Traditional Chinese Medicine, Shanghai, China

Corresponding author: Dr. Lujin Li; Center for Drug Clinical Evaluation, Shanghai University of Traditional Chinese Medicine, Shanghai, China; Email: lilujin666@163.com; Prof. Qingshan Zheng, Center for Drug Clinical Evaluation, Shanghai University of Traditional Chinese Medicine, Shanghai, China; Email: qingshan.zheng@drugchina.net 


\section{Abstract}

Background: In clinical practice, different chemotherapy schedules for NSCLC have potential impacts on overall survival, but not specific in NCCN guidelines. Although some meta-analyses have been conducted to identify the influencing factors, the results are limited by method. Our study is helpful to supplement the NCCN Guidelines.

Methods: In order to evaluate and quantify the potential relationship between demographic characteristics, disease characteristics, etc. and overall survival as well as their impact on objective response rate and safety, we developed parametric proportional hazard regression models to describe the overall survival of advanced NSCLC patients first-treated with the paclitaxel-platinum regimen.

Results: The principal finding of this research was that race significantly affected hazard of dying. Hazard of dying was 1.4 times higher in non-East Asians than in East Asians, and the hazard ratio of the OS curve was $0.71(95 \% \mathrm{CI}: 0.65-0.78)$. The median survival time in East Asians and non-East Asians were estimated as 12.2 (95\%CI: 10.5, 14.4) and 8.4 (95\%CI: 6.5. 11.0) months, respectively. The ORR was 37\% (95\%CI: 32 , $41)$ and 28\% (95\% CI: 25, 32) in East Asians and non-East Asians, respectively.

Conclusions: Paclitaxel-platinum regimen had different efficacy in two racial groups. The developed model suggests that the efficacy and safety of paclitaxel-platinum regimen can vary between different racial populations because of differences in influencing factors (doses, platinum type, cycles, etc.)

Keywords: Paclitaxel, Platinum, Advanced NSCLC, Overall Survival, Modeling and 
simulation

\section{Background}

Over the past decade, targeted therapy and immunotherapy have led to new developments in the treatment of non-small cell lung cancer (NSCLC) based on molecular and immunological research. ${ }^{1}$ Research in this area has shown that the incidence of NSCLC with EGFR mutation is the highest among various molecular subtypes. Approximately $20 \%$ of patients with advanced NSCLC have EGFR mutation, with ALK rearrangement, MET mutation, ROS1 rearrangement, and BRAF mutation showing a prevalence of approximately $5 \%, 4 \%, 1 \%$, and $2 \%$, respectively. ${ }^{2}$ However, more than $50 \%$ of patients with advanced NSCLC still have unknown gene mutations, and thus cannot receive targeted therapy. ${ }^{3}$ Lee (2014) compared targeted therapy and conventional chemotherapy in EGFR wild-type patients, and the results showed that conventional chemotherapy led to significantly higher progression-free survival (PFS) and objective response rate (ORR) than targeted therapy. ${ }^{4}$ In immunotherapy, patients with PD-L1 expression levels above 50\% could significantly benefit from conventional chemotherapy; however, in fact, only $23-28 \%$ of patients with advanced NSCLC had high PD-L1 expression levels. ${ }^{5}$ Therefore, most patients cannot benefit from targeted therapy or immunotherapy, and chemotherapy remains the cornerstone of treatment for advanced NSCLC.

The combination of paclitaxel and platinum drugs is a recognized first-line chemotherapy regimen for the treatment of advanced NSCLC. ${ }^{6}$ However, the NCCN 
Clinical Practice Guidelines in Oncology for NSCLC recommend conventional treatment options without clarifying the specific details, such as the times of administration, cycles of chemotherapy, and doses of paclitaxel in combination with platinum drugs, and thus may cause great differences between different paclitaxelplatinum regimens in clinical practice. Of particular concern is that the efficacy and safety of a paclitaxel-platinum regimen may not be identical in different populations. In a study by Soo (2011), Asians and Caucasians showed different responses to chemotherapy; Asians, who also received chemotherapy for advanced NSCLC, showed higher overall survival (OS) rates and longer median survival time than Caucasians. ${ }^{7}$ Consequently, finding a safer and more effective chemotherapy regimen for a paclitaxel-platinum regimen in different populations is not only beneficial for clinical practice but also a great help for the development of new drugs. In many clinical trials of new drugs, a paclitaxel-platinum regimen is often used as a positive control, basic therapy, and even external control for single-arm trials. Heterogeneity of treatment plans and patient populations in different trials may lead to differences in efficacy and safety. Therefore, when evaluating the sample size of new drugs in clinical trials, or when determining the cutoff value of efficacy or safety in external controls, caution should be taken to find the relevant literature data.

Gao (2012) found that paclitaxel had similar efficacy but better safety when administered once a week versus every 3 weeks, and the weekly schedule was suitable for elderly patients who could not tolerate the standard regimen and for patients with poor performance status scores. ${ }^{8}$ In terms of chemotherapy cycles, Soon (2009) found 
that longer cycles can statistically increase OS and PFS, but did not investigate whether the extension of the cycle would increase the incidence of adverse events. ${ }^{9}$ Moreover, this study was published relatively early, and only a few trials were included; thus, there are some potential limitations. Thus far, several studies have indicated that six cycles and three to four cycles of platinum-based first-line treatment did not significantly improve OS and PFS in patients with advanced NSCLC. In addition, the incidence of anemia was higher with six cycles; thus, fewer cycles of chemotherapy were recommended. ${ }^{10}$ It should be noted that these studies did not distinguish the heterogeneity of subjects in the trial, and thus their conclusions may be more prone to bias.

Model-based meta-analysis (MBMA) is a powerful analytical method for dealing with heterogeneity. Quantitative analysis of influencing factors can be conducted simultaneously by establishing a covariate model and obtaining quantitative results by evaluating the degree of influence of those factors on efficacy or safety. Importantly, MBMA can be used to analyze OS rate in the entire time course and not just in a single index, such as median survival time and 1-year survival rate, by establishing a proportional hazard regression model. After a hazard model is developed, it can be used to predict OS rate at any time within 5 years, such as $0.5,1,1.5$, and 2 years, showing better flexibility and practicability in the evaluation of new drugs.

In this study, MBMA was conducted to explore and quantitatively assess the influencing factors of paclitaxel-platinum regimens as a first-line chemotherapy in patients with advanced NSCLC, including cycles of chemotherapy, dosage, times of 
administration, and baseline characteristics of patients. We hope this research will offer a fresh perspective on the quantitative information necessary for clinical practice and provide a more accurate cutoff value for the distribution of positive drugs for clinical trials of new drugs.

\section{Patients and Methods}

\subsection{Literature sources}

A systematic literature search was performed using PubMed and the Cochrane Library and completed on July 1, 2019. The terms "carboplatin," "cisplatin," "paclitaxel," "albumin-bound paclitaxel," and "non-small cell lung cancer" were the key words used in the search. The search was limited to English language literature and clinical trials. Specific details on the search strategy are shown in the Supplementary.

\subsection{Patients, data extraction, and software}

All patients had advanced stage IIIB and IIIC or stage IV NSCLC and received carboplatin/cisplatin in combination with paclitaxel/albumin-bound paclitaxel as a firstline chemotherapy regimen. The patients had not received any treatment before enrollment, including surgical resection, radiotherapy, or chemotherapy. Specific inclusion and exclusion criteria are detailed in the Supplementary.

Excel (Microsoft, USA) was used to manage the literature characteristics, trial design, demographic characteristics, disease characteristics, efficacy, and safety results. The Engauge Digitizer (Mark Mitchell, USA) software was used to extract survival data 
from Kaplan-Meier curves in the literature.

NONMEM7.3 (ICON Development Solutions, USA) was used to construct the model and Bayesian feedback of model parameters in each experimental group. Model simulation and graph drawing were completed by R4.0.1 (Lucent Technologies, USA). Meta-analysis was performed using Stata Software Version 14 (2013; Stata Corp LP, College Station, TX, USA). $\mathrm{P} \leqslant 0.05$ indicated a statistically significant difference.

\subsection{Literature quality evaluation}

The Cochrane Risk of Bias Table was used to evaluate literature quality, including random sequence generation, allocation concealment, blinding of participants and personnel, blinding of outcome assessment, incomplete outcome data, selective reporting, and other biases. Other biases were defined as baseline characteristics that were not comparable between different groups in the trial.

\subsection{Modeling analysis of overall survival}

\subsubsection{Model development and model selection criteria}

In this study, a parametric proportional hazard regression model was used to explore OS data so as to analyze the distribution of OS time of patients treated with a paclitaxel-platinum regimen. The hazard function for death was based on the following equation:

Hazard function $h(t)=h_{0}(t) \cdot e^{\left(\alpha_{1} \cdot x_{1}+\alpha_{2} \cdot x_{2}+\cdots+\alpha_{n} \cdot x_{n}+\eta\right)}$

Exponential function

$$
h_{0}(t)=\lambda_{0}
$$


Gompertz function

Weibull function

Cumulative hazard function

Survival function

Standard error function

$$
h_{0}(t)=\lambda_{1} \cdot \exp \left(\beta_{1} \cdot t\right)
$$

OS rate is affected by many factors in survival analysis, and censored data are included in many studies. The distribution of OS was not exactly determined nor did it meet the normal distribution and homogeneity of variance. Therefore, hazard rate was used as a dependent variable in this study.

In formula (1), $h(t)$ represents the instantaneous hazard of dying at time $t$, which was obtained by multiplying two parts. Among them, $\mathrm{h}_{0}(\mathrm{t})$ describes the base hazard function, in which instantaneous hazard of dying changed over time. We first examined the fitting effectiveness of the exponential, Gompertz, and Weibull functions on survival data. In formulas (2)-(4), $\lambda_{0}$ and $\lambda_{1}$ represent the hazard rate at time 0 , and $\beta_{1}$ represents the regression coefficient of the hazard rate changing over time. In the exponential function, hazard rates were assumed to be constant values that did not change over time. Therefore, hazard rates were constant at each moment. However, in the Gompertz and Weibull functions, hazard rates would change over time as a function of exponential. $e^{\left(\alpha_{1} \cdot x_{1}+\alpha_{2} \cdot x_{2}+\cdots+\alpha_{n} \cdot x_{n}+\eta\right)}$ describe the effect of covariates on hazard rates, where $\mathrm{x}_{1}, \mathrm{x}_{2}, \ldots, \mathrm{x}_{\mathrm{n}}$ represent $1-\mathrm{n}$ covariates that affect hazard rates, and their impact coefficients on hazard rates are $\alpha_{1}, \alpha_{2}, \ldots, \alpha_{\mathrm{n}}$. In formula $(5), \Lambda(0, \mathrm{t})$ is the 
cumulative hazard rate from time 0 to $t$, which is the integral value of the hazard function $\mathrm{h}(\mathrm{t})$ from time 0 to $\mathrm{t}$. In formula $(6), \mathrm{S}(\mathrm{t})_{\mathrm{i}}$ is the survival function at time point $t$ for the i-th study, which was the reciprocal of the exponential cumulative hazard of dying $\left(\mathrm{e}^{-\Lambda(0, t)}\right)$. When $\mathrm{S}(\mathrm{t})_{\mathrm{i}}$ is $0.5, \mathrm{t}$ is the median survival time of the $\mathrm{i}$-th study. $\varepsilon_{i, t}$ is the residual variation in survival rate at time $t$ in the $i$-th study, and needs to be corrected by the standard error of the survival rate $\left(\mathrm{SE}_{\mathrm{i}, \mathrm{t}}\right)$ at that time point. That is, the larger the standard error, the greater the residual variation.

After establishing the base model, a covariate model was developed using the forward inclusion-backward elimination method. We screened out covariates that affected hazard of dying, including age, gender, performance status, clinical stage, pathological type, dosage, platinum type (carboplatin or cisplatin), cycles of chemotherapy, times of administration, and race. If the above covariates were not available in some studies, the median was used. As hazard of dying was the parameter, the inclusion and elimination procedures of all covariates were carried out on $\lambda$. The boundary values of forward inclusion and backward elimination were set to $\mathrm{P}<0.05$ and $\mathrm{P}<0.01$, respectively. Continuous variables were introduced as formula (8), and categorical variables were introduced as formula (9):

$$
\begin{aligned}
& \lambda_{0_{\text {individual }}}=\lambda_{0_{\text {typical }}} \times e^{\left(\eta+\left(\operatorname{COV}-\operatorname{COV}_{\text {median }}\right) \times \theta_{\text {COV }}\right)} \\
& \lambda_{0_{\text {individual }}}=\lambda_{0_{\text {typical }}} \times e^{\left(\eta+\operatorname{COV} \times \theta_{\text {COV }}\right)}
\end{aligned}
$$

In the covariate screening, the influence of a single covariate on the efficacy end point (OS) was investigated for primary screening. The detailed process is shown in Supplementary Table 1. If the objective function value of the model decreased by more 
than $3.84(\mathrm{df}=1, \mathrm{P}=0.05)$, the covariate was considered to have a significant influence on the parameters. Next, previously selected covariates were introduced forward and eliminated backward one by one to identify the covariates that had a significant influence on the model.

$\lambda_{\text {oindividual }}$ is the parameter value for hazard of dying in each study, and $\lambda_{\text {otypical }}$ is the typical value for hazard of dying in each study. COV is the covariate value of each study, $\mathrm{COV}_{\text {median }}$ is the median value of the covariate, and $\theta_{\mathrm{COV}}$ is the correction factor of the covariate to hazard rates. $\eta$ is the inter-trial variation of hazard rates. That is, the variation could not be explained by covariates.

\subsubsection{Model evaluation}

After the final model was established, the goodness-of-fit of the model was visually evaluated using the $\mathrm{R}$ (Lucent Technologies, USA) software, in which predicted values and residuals were checked to assess the potential bias of the model.

Next, sampling of the final model parameters was repeated 1000 times by Bootstrap to obtain the median and $95 \%$ confidence interval $(95 \% \mathrm{CI})$ of the model parameter distribution. They were then compared with the final model parameters to evaluate the stability of the model. Finally, we conducted 1000 simulations of the final model using the Monte Carlo method to obtain the median OS at each time and the $95 \% \mathrm{CI}$. Comparison of intervals with observations as well as visualization of the results were performed using the $\mathrm{R}$ software to determine whether the model is reliable and can accurately predict the results.

\subsubsection{Simulation}


After the final model was constructed, we found that some covariates significantly influenced OS in the screening process. The typical survival time curve and 95\%CI of

211 the covariate at different levels could be simulated based on the final model parameters.

212 We calculated the median survival time and survival rates within 5 years.

This study focused on the covariates (paclitaxel doses, platinum type, cycles of chemotherapy, and times of administration) related to the regimen. Even if the above factors were not found to significantly affect OS, the OS distribution of these covariates for different levels would be obtained through the following methods. The specific method was to obtain the model parameters of each experimental group using Bayesian feedback. Subsequently, to obtain the distribution of efficacy parameters at different covariate levels. That is, the point estimates of the model parameters and their $95 \%$ CIs. and survival rates within 5 years.

\subsection{Objective response rate and safety analysis}

The random-effects model in the single-arm meta-analysis was used to analyze ORR and the incidence of grade 3-4 adverse effects (AEs) in each experimental group 
according to the different covariate levels to obtain the typical values of ORR and the incidence of AEs at different covariate levels. Their $95 \%$ CIs were also obtained.

\section{Results}

\subsection{Data Characteristics}

In total, 31 articles were included. ${ }^{11-41} \mathrm{~A}$ total of 3,365 participants from 37 experimental groups were included in the analysis. The flow chart of the literature screening is shown in Figure 1. The baseline characteristics of the patients are shown in Table 1. The participants were all previously untreated patients with advanced or metastatic NSCLC who received a paclitaxel-platinum regimen as a first-line chemotherapy. Drugs were administered intravenously. The median age of these patients was 62.5 years (range 26-87 years), and most of them presented with advanced clinical stage IV (20.4\% stage IIIB and 79.6\% stage IV). Most patients $(98.3 \%)$ had an ECOG PS of 0-1. Regarding histology, squamous cell carcinoma accounted for $29 \%$ of cases, and adenocarcinoma accounted for $50 \%$. The proportions of other histology, such as large cell carcinoma and adenosquamous carcinoma, were minor. A total of $13.7 \%$ of the patients were from East Asia (including China, Japan, and South Korea). The median administered dose of paclitaxel was $175 \mathrm{mg} / \mathrm{m}^{2}\left(\right.$ range $\left.40-225 \mathrm{mg} / \mathrm{m}^{2}\right)$. The median administered dose of carboplatin was AUC $=6 \mathrm{mg} / \mathrm{mL} \cdot \min$ (range AUC $=2-6 \mathrm{mg} / \mathrm{mL} \cdot \mathrm{min}$ ). The median administered dose of cisplatin was $75 \mathrm{mg} / \mathrm{m}^{2}$ (range $20-200 \mathrm{mg} / \mathrm{m}^{2}$ ). In terms of chemotherapy cycles, $75.1 \%$ of patients received $4-6$ cycles, $57.4 \%$ received 6 cycles, and $15.9 \%$ received 4 cycles, with 21 -day and 28 -day cycles 
accounting for $88.4 \%$ and $2.7 \%$, respectively. Regarding the times of drug administration, $77.1 \%, 3 \%$, and $3.7 \%$ of patients were administered paclitaxel once, twice, and three times, respectively, and $76.6 \%, 3 \%$, and $4.2 \%$ of patients were administered platinum drugs once, twice, and three times, respectively. Regarding dosage forms, albumin-bound paclitaxel and paclitaxel liposomes were used in only two trials, and paclitaxel was in used the remaining trials. Among platinum drugs, cisplatin liposomes were used in only one trial, and cisplatin or carboplatin was used in the remaining trials.

\subsection{Model development and evaluation}

\subsubsection{Overall survival}

Analysis of pooled data from the 31 included trials revealed that the exponential hazard function had the best description of OS among the hazard function models, as it showed the most stable estimation of model parameters. Therefore, the exponential hazard function was chosen as the base hazard model. During covariate screening, we found that race had a significant influence on hazard of dying. The hazard of dying in non-East Asians was 1.4 times $\left(\mathrm{e}^{0.37}\right)$ higher than that in East Asians. The estimated values of the parameters of the final model are shown in Supplementary Table S5, and the covariate model is expressed as follows:

$$
h(t)=0.0572 \cdot e^{(\text {Race } \cdot 0.37)}
$$

In formula (10), Race 0 represents East Asians, and Race 1 represents non-East Asians. That is, the typical instantaneous hazard of dying in East Asians was 0.0572, 
and that in non-East Asians was $0.08281\left(0.0572 \cdot \mathrm{e}^{0.37}\right)$. The final model is as follows:

$$
P_{i}=e^{-\int_{0}^{t_{j}}\left(0.0572 \times e^{\left(N\left(0,0.245^{2}\right)+R A C E \times 0.37\right)}\right)}+\sqrt{\frac{P_{i, j}\left(1-P_{i, j}\right)}{N_{i, j}}} \times\left(N\left(0,1.916^{2}\right)\right)
$$

$P_{i}$ is the OS probability of the $\mathrm{i}$-th experimental group. $\mathrm{N}\left(0,0.245^{2}\right)$ is the intergroup variation, which satisfied the normal distribution of mean 0 and standard deviation $0.245 . \mathrm{N}\left(0,1.916^{2}\right)$ is the intragroup variation, which satisfied the normal distribution of mean 0 and standard deviation 1.916 .

After the final model was established, parameter values and standard errors were estimated by Bayesian feedback. Bootstrap was successfully minimized 1,000 times. The median of the model parameters estimated by Bootstrap and its $95 \% \mathrm{CI}$ were very close to the estimated parameters of the final model. This further indicates the robustness of our model. The model diagnostic plots are displayed in Supplementary Figure S2, which shows that the population predicted values versus observed values were relatively uniformly distributed around 0 . Although there were some dispersions compared with the individual predicted values, considering that the population predicted values contained intergroup variations, conditional weighted residuals were distributed between \pm 4 . In the plot of time versus conditional weighted residuals, distribution was slightly uneven, which may be related to the different visit times of each experimental group. These trials mainly focused on the visit point within 16 months. The VPC plot generated using the Monte Carlo method, as shown in Supplementary Figure S4, showed that most of the observed values fell within the $95 \% \mathrm{CI}$ of the predicted values. The results indicated the good predictive ability of the 
model. In summary, the model could accurately predict the OS probability of patients at each visit point.

The simulated results are shown in Figure 2, where the typical OS curve of East Asians was significantly different from that of non-East Asians. The $95 \%$ CIs of the respective typical OS curves did not overlap (hazard ratio, $0.71 ; 95 \% \mathrm{CI}, 0.65$ to 0.78 ). It was estimated that the median survival time in East Asians and non-East Asians was $12.2(95 \% \mathrm{CI}: 10.5,14.4)$ and $8.4(95 \% \mathrm{CI}: 6.5 .11 .0)$ months, respectively. As shown in Table 2, 1-year OS rates in East Asians and non-East Asians were 50.3\% (95\%CI: 45.0, 56.1) and 37.2\% (95\%CI: 27.3. 46.2), respectively. The estimated OS rates at other times are shown in Table 3.

Among the covariates predicted to be related to the regimen, none was found to have a significant effect on OS in the final model; however, OS curve for different schedules could still be obtained according to the distribution of model parameters under different schedules. As race had a significant effect on OS, to avoid the effect of heterogeneity on the results, we conducted a subgroup analysis based on race. Because of the complexity of race, all patients were divided into two major groups according to region: East Asians and non-East Asians. We explored the effects of four cycles of chemotherapy versus six cycles; 21 days per cycle versus 28 days; platinum drugs administered 1, 2, or 3 times per cycle; paclitaxel administered 1, 2, or 3 times per cycle; paclitaxel doses; and carboplatin versus cisplatin on OS in the two racial groups. The results of this analysis are presented in Figure 2. In Non-East Asians, six cycles of chemotherapy versus four cycles; 21 days per cycle versus 28 days; paclitaxel 200-225 
$\mathrm{mg} / \mathrm{m}^{2}$ versus $175 \mathrm{mg} / \mathrm{m}^{2}$; and paclitaxel administered once per cycle versus three times significantly increased OS time. OS was significantly longer with 21 days per cycle than with 28 days per cycle. One-time paclitaxel administration per cycle led to significantly longer OS than three-time paclitaxel administration per cycle. The same trends were observed in East Asians. However, the trends were not obvious owing to the small sample size of the experimental groups and the large variation between trials. In addition, one interesting result emerged that carboplatin tended to be more effective than cisplatin in East Asians, but showed no obvious tendency in non-East Asians. as not overlapping 95\%CIs. According to the meta-analysis, the ORR was $37 \%$ (95\% analysis of ORR. However, as shown in Figure 3A-B, there was no significant difference in ORR between East Asians and non-East Asians at different covariate levels in the subgroup metaMeta-analysis of ORR between the two racial groups showed that ORR in East Asians was significantly higher than that in non-East Asian patients, which manifested 32, 41) and 28\% (95\%CI: 25, 32) in East Asians and non-East Asians, respectively.

\subsubsection{Adverse effects}

The meta-analysis results of grade 3-4 AEs in the two racial groups are shown in Supplementary Table S6. In hematological toxicity analysis, there was a significant difference between the two racial groups in the incidences of leukopenia, neutropenia, 
and febrile neutropenia. The incidences of these AEs in East Asians and non-East Asians were 27\% (95\% CI: 17, 37) and 11\% (95\% CI: 8, 15), 54\% (95\% CI: 41, 67) and $19 \%(95 \%$ CI: 14,25$)$, and 20\% (95\% CI: 6, 51) and 3\% (95\% CI: 1, 6), respectively. Among non-hematological toxicities, only nervous system disease was significantly different between the two racial groups; its incidence was 3\% $(95 \% \mathrm{CI}: 1,5)$ in East Asians and 9\% $(95 \% \mathrm{CI}: 6,13)$ in non-East Asians. Figure 3C-F presents the metaanalysis results of grade 3-4 AEs in the subgroups. There were significant differences in the incidence of AEs between the two racial groups at different covariate levels. In both racial groups, the incidence of leukopenia was significantly higher following six cycles of chemotherapy than after four cycles of chemotherapy. Moreover, the incidence of leukopenia with 28 days per cycle was higher than that with 21 days per cycle in non-East Asians. The incidence of neutropenia following one-time paclitaxel administration per cycle was higher than that after three-time administration. Neutropenia incidence was also different between 28 days per cycle and 21 days per cycle. In East Asians, there were differences in the incidence of leucopenia between cycles of chemotherapy and between platinum types.

\section{Discussion}

In reviewing the literature, little data were found on the association between OS and the different influencing factors (doses, platinum type, cycles of chemotherapy, times of administration). One of the aims of this study was to simulate the relationship between OS and different influencing factors in the two racial groups (East Asians and 
non-East Asians) treated with a paclitaxel-platinum-based regimen, as well as the relationship between ORR, grade 3/4 AEs, and these influencing factors. The most obvious finding of the analysis was that the efficacy end point (OS) in non-East Asian patients was related to cycles of chemotherapy, duration (days) of one cycle, doses, and times of paclitaxel administration. The efficacy end point (OS) was related to platinum type in East Asians. Another important finding was that the incidence of leukopenia was related to cycles of chemotherapy and duration (days) of one cycle, and that neutropenia was related to the times of paclitaxel administration and duration (days) of one cycle in non-East Asian patients. The incidence of leukopenia in East Asian patients was related to cycles of chemotherapy and platinum type.

Our results showed that non-East Asians achieved longer OS when administered paclitaxel $200-225 \mathrm{mg} / \mathrm{m}^{2}$ once every 3 weeks for six cycles of chemotherapy. In contrast, the incidence of leukopenia was significantly higher with six cycles than with four cycles of chemotherapy. And the incidence of leukopenia was significantly higher with 28 days per cycle than with 21 days per cycle. The incidence of neutropenia was significantly higher when paclitaxel was administered once compared with when it was administered three times. Meanwhile, there were considerable differences in the incidence of neutropenia between 21 days per cycle and 28 days per cycle. This may occur because, owing to the lack of data on neutropenia, the confidence intervals were not completely separated and the difference was not significant. Sakakibara (2009) and Belani $(2008)^{42,43}$ reported that all efficacy parameters were similar between weekly and the standard every-3-week administration of carboplatin combined with paclitaxel. 
Their results were in accordance with our current findings, indicating that three-time paclitaxel administration was less toxic than but had comparable efficacy to one-time administration. This intriguing result could be attributed to the nonlinear pharmacokinetic behavior of paclitaxel. Paclitaxel systemic exposure is significantly increased as its systemic clearance is decreased, which changes its pharmacodynamics and increases the risk of systemic toxicity. ${ }^{44,45}$ Longer infusion of paclitaxel caused greater myelosuppression, but did not lead to higher response rate. ${ }^{46}$ In our study, as no data on infusion time were collected, it was impossible to verify whether infusion time affects the incidence of AEs. However, previous studies showed that there was no difference in OS between different infusion times.

In East Asians, carboplatin was more effective than cisplatin, but led to higher incidence of leukopenia. On the contrary, in a study by de Castria (2013) ${ }^{47}$, there was no significant difference in OS between cisplatin and carboplatin. It is possible that this previous finding was due to the lack of distinction between racial groups. In addition, our analysis revealed that four cycles of chemotherapy had better safety and lower incidence of leukopenia than six cycles of chemotherapy.

In the meta-analysis of ORR and the incidence of grade 3-4 AEs, meta-regression was used to explore the source of heterogeneity to avoid its influence. Further analysis showed that race, platinum type, and paclitaxel dose were the sources of heterogeneity in ORR, but there was no correlation between these factors. Race was the source of heterogeneity in the incidence of leucopenia and neutropenia. We then performed a subgroup analysis of ORR, leukopenia incidence, and neutropenia incidence in two 
racial groups. The results showed that the heterogeneity was significantly reduced, but still present. Further meta-regression revealed that platinum type was the source of heterogeneity in ORR among non-East Asian patients, and cycles of chemotherapy were the source of heterogeneity in leucopenia incidence. In addition, platinum type was the source of heterogeneity in leucopenia incidence among East Asians. The sources of heterogeneity, as identified by meta-regression, were consistent with the results of the meta-analysis. However, as meta-regression could not fully explain all heterogeneity, residual heterogeneity was still present.

Our analysis was based on the data provided by authors of the pooled studies, not on individual patient data. Despite our novel findings, there were several limitations to our study. First of all, because some studies did not indicate the race of patients, this study mainly divided two racial groups by region (East Asians and non-East Asians). In the sensitivity analysis, we found that after the data of Indians were deleted, the change rate of model parameter estimates was only $0.2 \%$. The results indicated that the stability of the model was not affected by it, and that these two racial groups could be considered equivalent to western and Asian populations. Second, considering that the efficacy of paclitaxel may be affected by the dosage forms, a related sensitivity analysis was also conducted in this study. Socinski (2012) and Tan (2018) noted that solvent paclitaxel and albumin-bound paclitaxel in combination with carboplatin prolonged OS in an elderly group, but the young group also showed similar results ${ }^{48,49}$. Our results indicated that after the data on albumin-bound paclitaxel and liposomal paclitaxel were deleted, the rate of change of model parameter estimates was $4 \%$, which was less than 
$5 \%$. Next, by comparing $95 \% \mathrm{CI}$ between the simulated efficacy and the original model, we found a high coincidence of these intervals. The most important result was that after the data on Indians, albumin-bound paclitaxel, and liposomal paclitaxel were deleted, race remained as the covariate that significantly affected OS. Our analysis confirms that compared with non-East Asians, East Asians had a better efficacy with paclitaxelplatinum regimen. We believe that the findings of this study will help most patients in NSCLC and be helpful to supplement the NCCN Guidelines. At the same time, we suggest that later studies should focus on the differences in efficacy between races.

\section{Conclusion}

This study aimed to develop a model for quantitative analysis of the effects of different covariates on the OS of previously untreated advanced NSCLC patients treated with a paclitaxel-platinum-based regimen. The results showed that the paclitaxel-platinum-based regimen had different efficacy in two racial groups, namely Non-East Asians and East Asians. Therefore, this regimen should be adjusted accordingly when used clinically. East Asians had longer OS and higher 5-year OS rate after treatment with a paclitaxel-platinum-based regimen than non-East Asians. Moreover, the ORR significantly improved. However, the incidence of AEs was relatively high in the East Asian population owing to low tolerance.

\section{Abbreviations}

AEs, adverse effects; 95\%CI, 95\% confidence interval; ECOG PS, Eastern Cooperative 
461 progression-free survival.

\author{
Not applicable
}

\section{Consent for publication}

Not applicable

\section{Competing interests}

\section{Funding:} 2018ZX10303501).

Oncology Group performance status; MBMA, model-based meta-analysis; NSCLC, non-small cell lung cancer; ORR, objective response rate; OS, overall survival; PFS,

\section{Ethics approval and consent to participate}

\section{Availability of data and materials}

All data generated or analysed during this study are included in this published article and its supplementary information files.

The authors have declared that no competing interests exist

This study was provided by the project of Shanghai S\&T Innovation Plan (17401970900) and National Major S\&T Project (2018ZX09734005, 2018ZX09711001-009-011, 2018ZX09731016, 2017ZX09304003, and 
474 Authors' contributions

475 Chenyang Zhao conceived the study and drafted the manuscript. All authors revised the 476 manuscript. All authors read and approved the final manuscript.

\section{Acknowledgements}

The authors acknowledge Dr. Qianyu Yang, Lin Zhu, Dr. Xijun Zheng, Dr. Ningyuan

\section{References}

1. Huang J, Ding Z, Luo Q, Xu W. Cancer cell-derived exosomes promote cell proliferation and inhibit cell apoptosis of both normal lung fibroblasts and non-small cell lung cancer cell through delivering alpha-smooth muscle actin. American journal of translational research 2019;11:1711-23.

2. Arbour KC, Riely GJ. Systemic Therapy for Locally Advanced and Metastatic Non-Small Cell Lung Cancer: A Review. JAMA 2019;322:764-74.

3. Pao W, Girard N. New driver mutations in non-small-cell lung cancer. The Lancet Oncology 2011;12:175-80.

4. Lee JK, Hahn S, Kim DW, Suh KJ, Keam B, Kim TM, Lee SH, Heo DS. Epidermal growth factor receptor tyrosine kinase inhibitors vs conventional chemotherapy in non-small cell lung cancer harboring wild-type epidermal growth factor receptor: a meta-analysis. JAMA 2014;311:1430-7.

5. Reck M, Rodriguez-Abreu D, Robinson AG, Hui R, Csoszi T, Fulop A, Gottfried M, Peled N, Tafreshi A, Cuffe S, O'Brien M, Rao S, et al. Pembrolizumab versus Chemotherapy for PD-L1Positive Non-Small-Cell Lung Cancer. N Engl J Med 2016;375:1823-33.

6. Wang Q, Chen Y, Feng H, Zhang B, Wang H. Prognostic and predictive value of HURP in non-small cell lung cancer. Oncology reports 2018;39:1682-92.

7. Soo R, Loh M, Mok T, Ou S, Cho B, Yeo W, Tenen D, Soong R. Ethnic differences in survival outcome in patients with advanced stage non-small cell lung cancer: results of a meta-analysis of 
randomized controlled trials. Journal of thoracic oncology : official publication of the International Association for the Study of Lung Cancer 2011;6:1030-8.

8. Gao G, Chu H, Zhao L, Gui T, Xu Q, Shi J. A meta-analysis of paclitaxel-based chemotherapies administered once every week compared with once every 3 weeks first-line treatment of advanced non-small-cell lung cancer. Lung Cancer 2012;76:380-6.

9. Soon Y, Stockler M, Askie L, Boyer M. Duration of chemotherapy for advanced non-small-cell lung cancer: a systematic review and meta-analysis of randomized trials. Journal of clinical oncology : official journal of the American Society of Clinical Oncology 2009;27:3277-83.

10. Huang TC, Campbell TC. Comparison of weekly versus every 3 weeks paclitaxel in the treatment of advanced solid tumors: a meta-analysis. Cancer Treat Rev 2012;38:613-7.

11. Okuma Y, Hosomi Y, Takahashi S, Nakahara Y, Watanabe K, Nagamata M, Takagi Y, Mikura S. A phase II study of nanoparticle albumin-bound paclitaxel plus carboplatin as the first-line therapy in elderly patients with previously untreated advanced non-small cell lung cancer. Cancer chemotherapy and pharmacology 2016;78:383-8.

12. Reck M, Krzakowski M, Chmielowska E, Sebastian M, Hadler D, Fox T, Wang Q, Greenberg J, Beckman RA, von Pawel J. A randomized, double-blind, placebo-controlled phase 2 study of tigatuzumab (CS-1008) in combination with carboplatin/paclitaxel in patients with chemotherapynaive metastatic/unresectable non-small cell lung cancer. Lung Cancer 2013;82:441-8.

13. Ishii Y, Fujimoto S, Okazaki K, Miyoshi M, Furihata T, Hase I, Takizawa H, Kikkawa Y, Yamada I, Fukuda T. Fractionated administration of carboplatin/paclitaxel reduces neurotoxicity in patients with advanced non-small cell lung cancer. Anti-cancer drugs 2011;22:926-32.

14. Stathopoulos GP, Antoniou D, Dimitroulis J, Stathopoulos J, Marosis K, Michalopoulou P. Comparison of liposomal cisplatin versus cisplatin in non-squamous cell non-small-cell lung cancer. Cancer chemotherapy and pharmacology 2011;68:945-50.

15. Han B, Xiu Q, Wang H, Shen J, Gu A, Luo Y, Bai C, Guo S, Liu W, Zhuang Z, Zhang Y, Zhao Y, et al. A multicenter, randomized, double-blind, placebo-controlled study to evaluate the efficacy of paclitaxel-carboplatin alone or with endostar for advanced non-small cell lung cancer. J Thorac Oncol 2011;6:1104-9.

16. Jahnke K, Keilholz U, Luftner D, Thiel E, Schmittel A. Randomized phase II study of paclitaxel and carboplatin or vinorelbine in advanced non-small cell lung cancer. Anticancer research 2011;31:317-23.

17. Weissman $\mathrm{CH}$, Reynolds $\mathrm{CH}$, Neubauer MA, Pritchard S, Kobina S, Asmar L. A phase III randomized trial of gemcitabine-oxaliplatin versus carboplatin-paclitaxel as first-line therapy in patients with advanced non-small cell lung cancer. J Thorac Oncol 2011;6:358-64.

18. Arrieta O, Gonzalez-De la Rosa CH, Arechaga-Ocampo E, Villanueva-Rodriguez G, CeronLizarraga TL, Martinez-Barrera L, Vazquez-Manriquez ME, Rios-Trejo MA, Alvarez-Avitia MA, Hernandez-Pedro N, Rojas-Marin C, De la Garza J. Randomized phase II trial of All-trans-retinoic acid with chemotherapy based on paclitaxel and cisplatin as first-line treatment in patients with advanced non-small-cell lung cancer. J Clin Oncol 2010;28:3463-71.

19. Scagliotti G, Novello S, von Pawel J, Reck M, Pereira JR, Thomas M, Abrao Miziara JE, Balint B, De Marinis F, Keller A, Aren O, Csollak M, et al. Phase III study of carboplatin and paclitaxel alone or with sorafenib in advanced non-small-cell lung cancer. J Clin Oncol 2010;28:1835-42.

20. Heymach JV, Paz-Ares L, De Braud F, Sebastian M, Stewart DJ, Eberhardt WE, Ranade AA, Cohen G, Trigo JM, Sandler AB, Bonomi PD, Herbst RS, et al. Randomized phase II study of vandetanib 
alone or with paclitaxel and carboplatin as first-line treatment for advanced non-small-cell lung cancer. J Clin Oncol 2008;26:5407-15.

21. Chen YM, Perng RP, Tsai CM, Whang-Peng J. A Phase II randomized study of paclitaxel plus carboplatin or cisplatin against chemo-naive inoperable non-small cell lung cancer in the elderly. J Thorac Oncol 2006;1:141-5.

22. Giorgio CG, Pappalardo A, Russo A, Giuffrida D, Santini D, Petralia G, Castorina S, Nunzio R, Failla G, Bordonaro R. A phase II study of carboplatin and paclitaxel as first line chemotherapy in elderly patients with advanced non-small cell lung cancer (NSCLC). Lung Cancer 2006;51:357-62.

23. Ichiki M, Kawasaki M, Takayama K, Ninomiya K, Kuba M, Iwami F, Miyazaki N, Oishi K, Takeo S, Aizawa H, Nakanishi Y. A multicenter phase II study of carboplatin and paclitaxel with a biweekly schedule in patients with advanced non-small-cell lung cancer: Kyushu thoracic oncology group trial. Cancer chemotherapy and pharmacology 2006;58:368-73.

24. Williamson SK, Crowley JJ, Lara PN, Jr., McCoy J, Lau DH, Tucker RW, Mills GM, Gandara DR. Phase III trial of paclitaxel plus carboplatin with or without tirapazamine in advanced non-smallcell lung cancer: Southwest Oncology Group Trial S0003. J Clin Oncol 2005;23:9097-104.

25. Herbst RS, Prager D, Hermann R, Fehrenbacher L, Johnson BE, Sandler A, Kris MG, Tran HT, Klein P, Li X, Ramies D, Johnson DH, et al. TRIBUTE: a phase III trial of erlotinib hydrochloride (OSI-774) combined with carboplatin and paclitaxel chemotherapy in advanced non-small-cell lung cancer. J Clin Oncol 2005;23:5892-9.

26. Chen CH, Chang WC, Lin MC, Hsu JW, Chao TY, Tsao TC. Phase II study of paclitaxel (Genaxol) and cisplatin combination in treating Chinese patients with advanced non-small cell lung cancer (NSCLC). Lung Cancer 2002;38:91-6.

27. Ogawara M, Kawahara M, Hosoe S, Atagi S, Kawaguchi T, Okishio K, Naka N, Sunami T, Mitsuoka $\mathrm{S}$, Inoue K, Haryu H, Yoneda T, et al. A feasibility study of paclitaxel $225 \mathrm{mg} / \mathrm{m}(2)$ and carboplatin $\mathrm{AUC}=6$ in untreated advanced non-small cell lung cancer patients in Japan. Japanese journal of clinical oncology 2002;32:48-53.

28. Bonomi P, Kim K, Fairclough D, Cella D, Kugler J, Rowinsky E, Jiroutek M, Johnson D. Comparison of survival and quality of life in advanced non-small-cell lung cancer patients treated with two dose levels of paclitaxel combined with cisplatin versus etoposide with cisplatin: results of an Eastern Cooperative Oncology Group trial. J Clin Oncol 2000;18:623-31.

29. Helsing M, Thaning L, Sederholm C, Lamberg K, Martinsson JE, Ek L, Månsson T, Andersson L, Hero U, Anjedani D, Svennson G. Treatment with paclitaxel 1-h infusion and carboplatin of patients with advanced non-small-cell lung cancer: a phase II multicentre trial. Joint Lung Cancer Study Group. Lung Cancer 1999;24:107-13.

30. Digumarti R, Wang Y, Raman G, Doval D, Advani S, Julka P, Parikh P, Patil S, Nag S, Madhavan $\mathrm{J}$, et al. A randomized, double-blind, placebo-controlled, phase II study of oral talactoferrin in combination with carboplatin and paclitaxel in previously untreated locally advanced or metastatic non-small cell lung cancer Journal of thoracic oncology, vol. 6, 2011:1098 - 103.

31. Pathak A, Bhutani M, Guleria R, Bal S, Mohan A, Mohanti B, Sharma A, Pathak R, Bhardwaj N, Prasad K, et al. Chemotherapy alone vs. chemotherapy plus high dose multiple antioxidants in patients with advanced non small cell lung cancer Journal of the american college of nutrition, vol. 24, 2005:16 - 21.

32. Gao J, Guan S, Deng W, Qiu G, Rao Z, Zhang B. Clinical study of comparing LP and TP regimens in the treatment of advanced non-small cell lung cancer Chinese-german journal of clinical 
oncology, vol. 9, 2010:198 - 200.

33. Kim SW, Suh C, Lee SD, Kim WS, Kim DS, Kim WD, Lee JS. Weekly low dose paclitaxel and cisplatin as first-line chemotherapy for advanced non-small cell lung cancer. Lung Cancer 2003;41:221-6.

34. Sweeney C, Zhu J, Sandler A, Schiller J, Belani C, Langer C, Krook J, Harrington D, Johnson D. Outcome of patients with a performance status of 2 in Eastern Cooperative Oncology Group Study E1594: a phase III trial in patients with metastatic nonsmall cell lung carcinoma Cancer, vol. 92, 2001:2639 - 47.

35. Belani CP, Chakraborty BC, Modi RI, Khamar BM. A randomized trial of TLR-2 agonist CADI-05 targeting desmocollin-3 for advanced non-small-cell lung cancer. Annals of oncology : official journal of the European Society for Medical Oncology 2017;28:298-304.

36. Rozzi A, Nardoni C, Corona M, Restuccia MR, Falbo T, Lanzetta G. Weekly regimen of paclitaxel and carboplatin as first-line chemotherapy in elderly patients with stage IIIB-IV non small cell lung cancer (NSCLC): results of a phase II study. Journal of chemotherapy (Florence, Italy) 2010;22:419-23.

37. Blumenschein GR, Jr., Khuri FR, von Pawel J, Gatzemeier U, Miller WH, Jr., Jotte RM, Le Treut J, Sun SL, Zhang JK, Dziewanowska ZE, Negro-Vilar A. Phase III trial comparing carboplatin, paclitaxel, and bexarotene with carboplatin and paclitaxel in chemotherapy-naive patients with advanced or metastatic non-small-cell lung cancer: SPIRIT II. J Clin Oncol 2008;26:1879-85.

38. Lilenbaum RC, Chen CS, Chidiac T, Schwarzenberger PO, Thant M, Versola M, Lane SR. Phase II randomized trial of vinorelbine and gemcitabine versus carboplatin and paclitaxel in advanced nonsmall-cell lung cancer. Annals of oncology : official journal of the European Society for Medical Oncology 2005;16:97-101.

39. Hainsworth JD, Urba WJ, Hon JK, Thompson KA, Stagg MP, Hopkins LG, Thomas M, Greco FA. One-hour paclitaxel plus carboplatin in the treatment of advanced non-small cell lung cancer: results of a multicentre, phase II trial. European journal of cancer (Oxford, England : 1990) 1998;34:6548.

40. Chen K, Lin Q, Chang C, Zhao Y, Liu Y, Wang N, Su H, Huang Y. A clinical comparative study of GP and TP 1st-line chemotherapies for advanced non-small cell lung cancer. Chinese-german journal of clinical oncology 2010;9:621 - 24.

41. Wheatley-Price P, Gadgeel S, Takahashi T, Li X, Dar M, Blumenschein GR, Jr. Phase 1b/2 Randomized Study of MEDI-575 in Combination With Carboplatin Plus Paclitaxel Versus Carboplatin Plus Paclitaxel Alone in Adult Patients With Previously Untreated Advanced NonSmall-Cell Lung Cancer. Clinical lung cancer 2019;20:e362-e68.

42. Sakakibara T, Inoue A, Sugawara S, Maemondo M, Ishida T, Usui K, Abe T, Kanbe M, Watanabe H, Saijo Y, Nukiwa T. Randomized phase II trial of weekly paclitaxel combined with carboplatin versus standard paclitaxel combined with carboplatin for elderly patients with advanced non-smallcell lung cancer. Annals of oncology : official journal of the European Society for Medical Oncology 2010;21:795-99.

43. Belani CP, Ramalingam S, Perry MC, LaRocca RV, Rinaldi D, Gable PS, Tester WJ. Randomized, phase III study of weekly paclitaxel in combination with carboplatin versus standard every-3-weeks administration of carboplatin and paclitaxel for patients with previously untreated advanced nonsmall-cell lung cancer. J Clin Oncol 2008;26:468-73.

44. Sparreboom A, van Tellingen O, Nooijen W, Beijnen J. Nonlinear pharmacokinetics of paclitaxel in 
mice results from the pharmaceutical vehicle Cremophor EL. Cancer research 1996;56:2112-5.

45. Marupudi NI, Han JE, Li KW, Renard VM, Tyler BM, Brem H. Paclitaxel: a review of adverse toxicities and novel delivery strategies. Expert Opin Drug Saf 2007;6:609-21.

46. Eisenhauer E, ten Bokkel Huinink W, Swenerton K, Gianni L, Myles J, van der Burg M, Kerr I, Vermorken J, Buser K, Colombo N. European-Canadian randomized trial of paclitaxel in relapsed ovarian cancer: high-dose versus low-dose and long versus short infusion. Journal of clinical oncology : official journal of the American Society of Clinical Oncology 1994;12:2654-66.

47. de Castria TB, da Silva EM, Gois AF, Riera R. Cisplatin versus carboplatin in combination with third-generation drugs for advanced non-small cell lung cancer. Cochrane Database Syst Rev 2013:CD009256.

48. Socinski MA, Bondarenko I, Karaseva NA, Makhson AM, Vynnychenko I, Okamoto I, Hon JK, Hirsh V, Bhar P, Zhang H, Iglesias JL, Renschler MF. Weekly nab-paclitaxel in combination with carboplatin versus solvent-based paclitaxel plus carboplatin as first-line therapy in patients with advanced non-small-cell lung cancer: final results of a phase III trial. J Clin Oncol 2012;30:205562.

49. Tan H, Hu J, Liu S. Efficacy and safety of nanoparticle albumin-bound paclitaxel in non-small cell lung cancer: a systematic review and meta-analysis. Artif Cells Nanomed Biotechnol 2019;47:26877. 
Records identified through database searching $(\mathrm{n}=2284)$

- PubMed $(\mathrm{n}=843)$

- Cochrane Library $(\mathrm{n}=1441)$
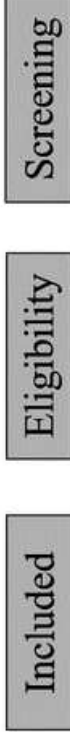

Studies included in quantitative synthesis $(n=31)$
Duplicates excluded $(\mathrm{n}=216)$

Titles and abstracts excluded $(\mathrm{n}=901)$

- Quality of life analyses $(n=22)$

- Not non-small cell lung cancer $(n=54)$

- Not the combination of paclitaxel and platinum $(\mathrm{n}=235)$

- Second-line chemotherapy $(n=24)$

- Neoadjuvant or Adjuvant Chemotherapy $(n=12)$

- Maintenance Treatment $(\mathrm{n}=18)$

- Concomitant with radiotherapy or immunotherapy or targeted therapy $\mathrm{n}=(536)$

Full-text articles excluded $(\mathrm{n}=1136)$

- Not clinical trials $(\mathrm{n}=211)$

- Not in stage $3 B$ or $4(n=178)$

- Patients had been previously treated in surgery, radiotherapy or chemotherapy $(n=224)$

- Dose escalation not fixed $(n=29)$

- Repeated studies $(n=64)$
- No full-text articles ( $n=385)$

- Not in English $(n=14)$

- No efficacy data $n=(18)$

- No inclusion and exclusion criteria $n=(13)$

\section{Figure 1}

Flow chart of the systematic reviews' inclusion. 

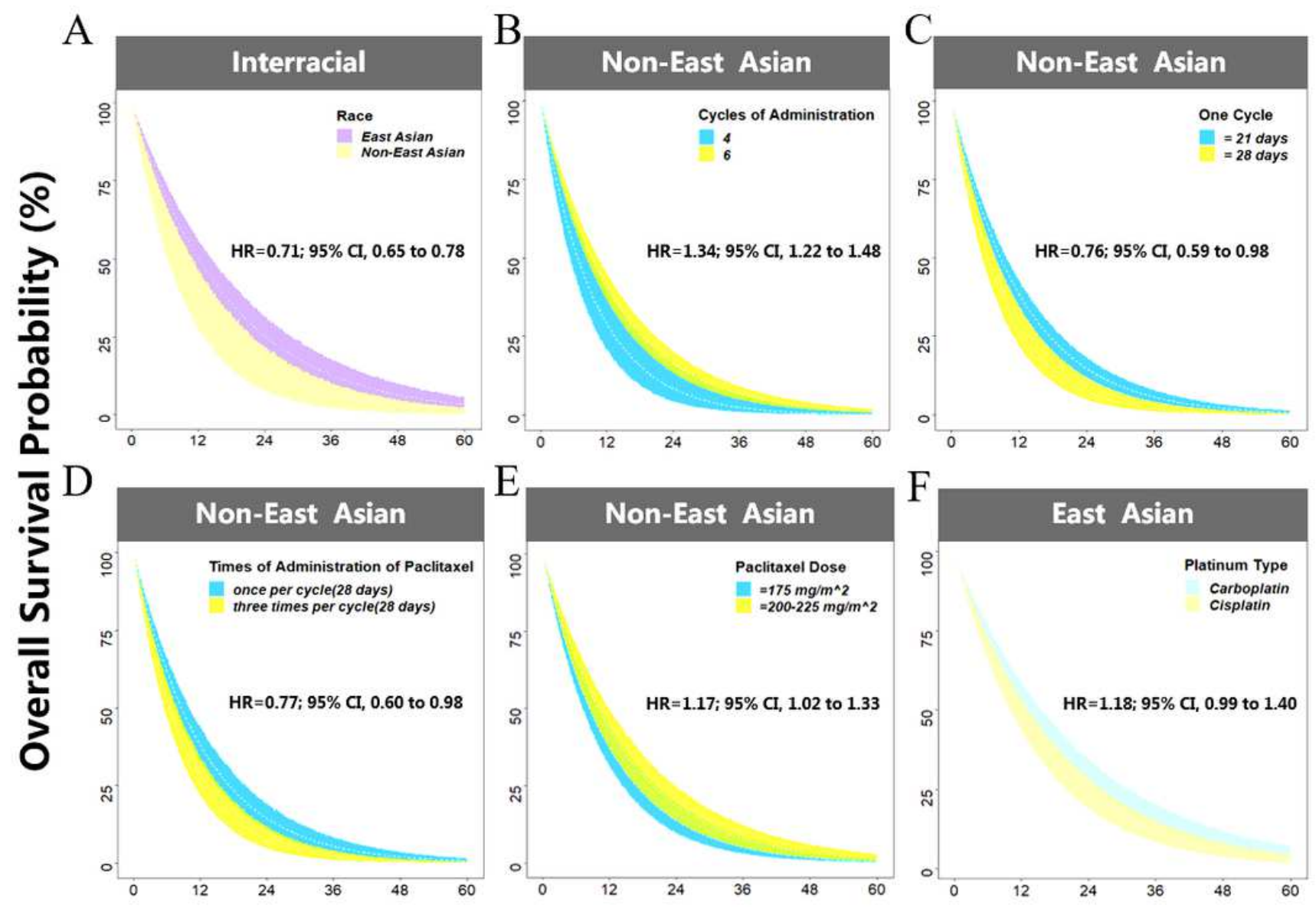

\section{Time (month)}

\section{Figure 2}

Predicted OS curve (and its $95 \% \mathrm{Cl}$ ) of different racial groups and typical schedules. The shadow represents $95 \% \mathrm{Cls}$ of OS. The dotted line represents model-predicted 50 th percentiles of OS. (A) East Asians versus Non-East Asians (B) 4 versus 6 cycles of chemotherapy in Non-East Asian (C) 21 days per cycle versus 28 days in Non-East Asian (D) Paclitaxel administered 1 versus 3 times per cycle in Non-East Asian (E) Paclitaxel 175 mg/m2 versus 200-225 mg/m2 in Non-East Asian; (F) Carboplatin versus cisplatin in East Asian. 
A Objective Response Rate (Non-East Asian)

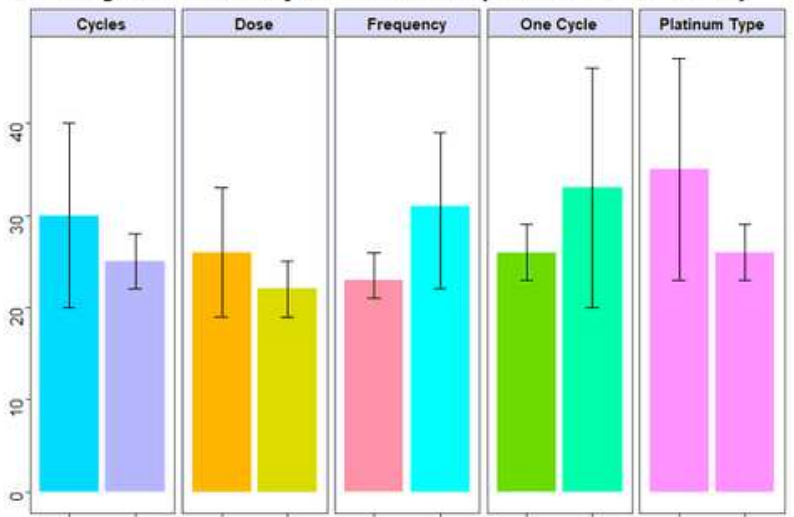

$\mathrm{C}$

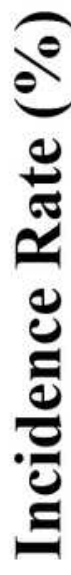

$\mathrm{B}$

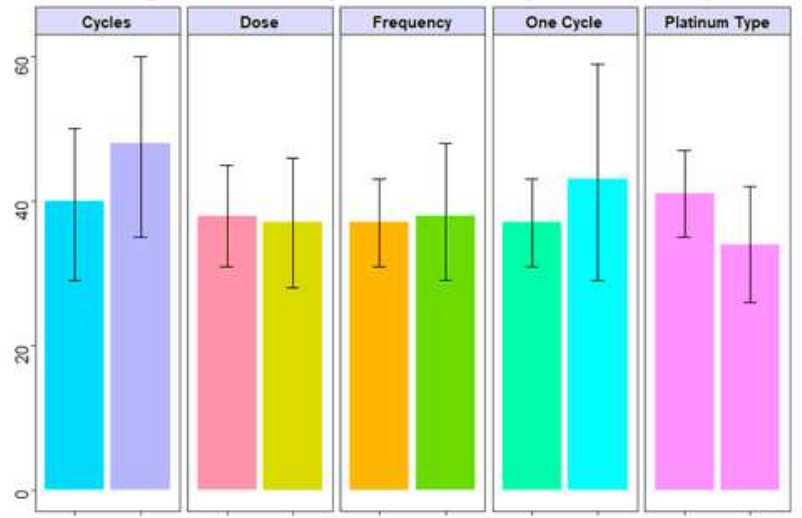

D Leukopenia grade3,4 (East Asian)

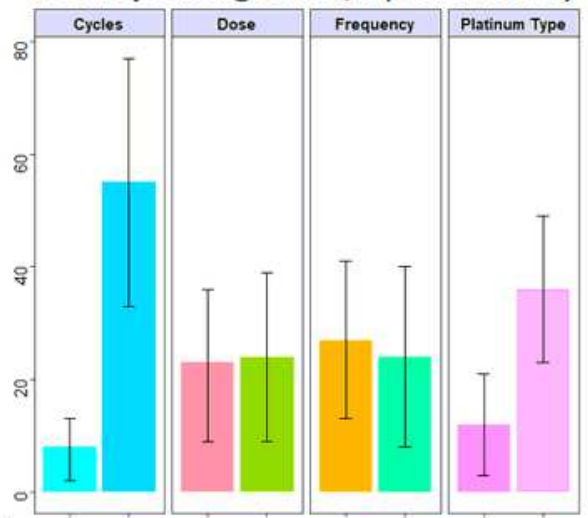

F Neutropenia grade3,4 (East Asian)

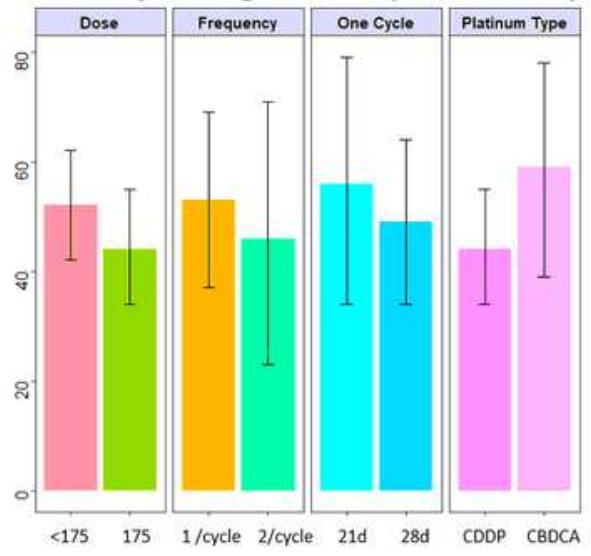

Covariate

\section{Figure 3}

Visualization results of ORR and grade 3-4 AEs at different covariate levels in subgroup meta-analysis. Error bars represent $95 \% \mathrm{Cl}$ based on meta-analysis. Cycles, cycles of chemotherapy; Dose, paclitaxel doses, Frequency, times of administration of paclitaxel; One cycle, duration (days) of one cycle;21d, 21 days; 28d, 28days; CDDP, cisplatin; CBDCA, carboplatin. Different covariate levels were divided into cycles of chemotherapy (4 versus 6), paclitaxel dose (175 mg/m2versus $200-225 \mathrm{mg} / \mathrm{m} 2,<175 \mathrm{mg} / \mathrm{m} 2$ 
versus $175 \mathrm{mg} / \mathrm{m} 2)$, times of administration of paclitaxel(1/cycle versus $3 /$ cycle, $1 /$ cycle versus $2 /$ cycle), duration (days) of one cycle(21days versus 28 days), platinum type(cisplatin versus carboplatin).

\section{Supplementary Files}

This is a list of supplementary files associated with this preprint. Click to download.

- Table1.pdf

- Table2.pdf

- Table3.pdf

- Supplementary.docx 